\title{
Révisionnisme stœchiométrique
}

\author{
Rédacteur en Chef, \\ Inserm U. 426 et \\ Département de Physiologie, \\ UFR de Médecine Xavier \\ Bichat, BP 416, \\ 16, rue Henri Huchard, \\ 75870 Paris Cedex 18, France. \\ gf@bichat.inserm.fr
} électrochimique favo-

$>$ La tendance est commune en biologie, comme dans d'autres domaines scientifiques probablement (pour ne parler que de ceux-là...), de rebâtir le monde autour de la dernière découverte en date. Ce faisant, les traces du passé, parfois gênantes, pour un peu s'estomperaient. Une lecture en perspective des éditions successives d'un traité de médecine est, à cet égard, une intéressante illustration. Depuis un quart de siècle, un traité de néphrologie internationalement reconnu met à jour, tous les cinq ans, les connaissances de cette discipline, dont la croissance explosive est un exemple du dynamisme de la recherche biomédicale. Le point y est fait, entre autres sujets, sur le transport du phosphate par l'épithélium rénal, depuis les mécanismes qui le soustendent jusqu'à sa pathologie.

Lors de la parution de la première édition, en 1976, l'approche des transports tubulaires rénaux sur des fractions cellulaires est balbutiante [1]. L'essentiel des connaissances repose alors sur des expériences de clairances (recueils minutés d'urine et de plasma) et de microponctions tubulaires. On avait démontré que la réabsorption de phosphate par le tubule rénal, tubule que l'on savait déjà également disséquer et microperfuser in vitro, dépend de la présence de sodium dans la lumière tubulaire. L'idée d'un co-transport sodiumphosphate, un $\mathrm{NaPi}$, était née.

Les preuves fonctionnelles de l'existence d'un tel cotransport sont rapportées cinq ans plus tard dans la deuxième édition [2]. L'utilisation de vésicules de bordures en brosse, fragments de membranes apicales de cellules tubulaires proximales refermées sur ellesmêmes, a permis à plusieurs groupes d'asseoir les bases du transport tubulaire de phosphate : cet ion entre dans les cellules, depuis la lumière, grâce à un système de co-transport sodium-phosphate qui utilise le gradient rable à l'entrée de sodium pour faire pénétrer le phosphate. Un ion phosphate divalent $\mathrm{HPO}_{4}{ }^{2-}$ est transporté en compagnie de deux ions sodium $\mathrm{Na}^{+}$; la stœchiométrie est de 2/1 ; le $\mathrm{NaPi}$ est électroneutre.

Ces caractéristiques sont reprises cinq ans plus tard dans la troisième édition du traité, appuyées par de nouvelles études expérimentales qui confirment l'électroneutralité du NaPi tubulaire rénal [3]. Comme dans les éditions précédentes, il est également souligné que la luxuriance de l'expérimentation visant à élucider le mécanisme d'entrée apicale du phosphate dans les cellules tubulaires contraste avec la pauvreté des connaissances sur les voies de sortie basolatérale du phosphate des cellules vers le sang.

L'édition suivante (nous sommes en 1991) trouve les choses en l'état [4]. Tout juste est-il fait mention de l'éventualité, en conditions très acides et non physiologiques, d'un possible transport de phosphate monovalent avec du sodium, toujours selon une stœchiométrie 2/1 (deux ions sodium pour un ion phosphate). On commence néanmoins à s'interroger sur la nature moléculaire de ce $\mathrm{NaPi}$ apical. Les premières pistes, évoquées alors, se refroidissent vite et c'est le groupe de Heini Murer, à Zurich (Suisse), qui acquiert, très rapidement, une position de quasi-monopole sur la biologie moléculaire du transport rénal de phosphate. Si, dans un premier temps, les données obtenues sur bordures en brosse coexistent avec les découvertes moléculaires récentes [5], la situation bascule à la fin des années 1990. Le NaPi majoritaire dans le rein, appelé NPT2a, est cloné : sa séquence est élucidée dans plusieurs espèces. Sa détection par des anticorps spécifiques confirme sa localisation dans la bordure en brosse ; son expression est modulée par l'hormone parathyroïdienne et l'apport alimentaire de phosphate. Bref, c'est $L \varepsilon$ système de transport que l'on cher- 
chait... Seule ombre au tableau, les données fonctionnelles issues de l'expression hétérologue de la protéine clonée dans des ovocytes de xénope, loin de confirmer les résultats antérieurs obtenus sur des vésicules de bordure en brosse, font état d'un transport électrogénique, de stœchiométrie $3 / 1$, faisant entrer ensemble un ion phosphate $\mathrm{HPO}_{4}{ }^{2-}$ et trois ions $\mathrm{Na}^{+}$.

Comme par enchantement, les bordures en brosse disparaissent alors du champ d'intérêt et ne sont même plus mentionnées $[6,7]$. Nous sommes passés de la « vérité » du rein à la « vérité » du modèle qui, un temps, occupe tout l'espace, sans partage.

II n'y a que quelques mois qu'une équipe de chercheurs japonais a découvert un nouveau NaPi rénal [8]. Lui aussi est localisé dans les bordures en brosse. Son expression y est très intense, au moins au cours de la croissance. Exprimé dans des ovocytes de xénope, il assure un co-transport sodium-phosphate... électroneutre, de stœchiométrie 2/1. La suite dira si c'est ce $\mathrm{NaPi}$-là qui confère aux membranes rénales leurs caractéristiques, nécessaire résultante de l'activité de l'ensemble des protéines qui y sont exprimées.

Peut-être ce sésame ouvrira-t-il à nouveau la porte aux bordures en brosse dans la vaste collection des « matériels et méthodes ». Elles se feront discrètes, c'est promis, pour ne pas porter ombrage aux étoiles du moment. Elles ne sont que la mémoire. $\diamond$

Stoichiometric revisionism

\section{RÉFÉRENCES}

1. Goldberg M, Agus ZS, Goldfarb S. Renal handling of phosphate, calcium, and magnesium. In : Brenner $B M$, Rector FC, eds. The kidney. Philadelphie: W.B. Saunders Company, 1976: 344-90.

2. Sutton RAL, Dirks JH. Renal handling of phosphate, calcium, and magnesium. In : Brenner BM, Rector FC, eds. The kidney, $2^{\mathrm{e}}$ ed. Philadelphie : W.B. Saunders Company, 1981 : 551-618.

3. Knochel JP, Jacobson HR. Renal handling of phosphorus, clinical hypophosphatemia, and phosphorus deficiency. In : Brenner BM, Rector FC, eds. The kidney, $3^{e}$ ed. Philadelphie : W.B. Saunders Company, 1986 : 619-62.

4. Suki WN, Rouse D. Renal transport of calcium, magnesium, and phosphorus. In : Brenner BM, Rector FC, eds. The kidney, $4^{\mathrm{e}} \mathrm{ed}$. Philadelphie: W.B. Saunders Company, 1991 : 380-423.
5. Suki WN, Rouse D. Renal transport of calcium, magnesium, and phosphate. In : Brenner BM, ed. Brenner and Rector's the kidney, $5^{\mathrm{e}} \mathrm{ed}$.

Philadelphie : W.B. Saunders Company, 1996 : 472-515.

6. Suki WN, Lederer ED, Rouse D. Renal transport of calcium, magnesium, and phosphate. In : Brenner BM, ed. Brenner and Rector's the kidney, $6^{\mathrm{e}}$ ed.

Philadelphie : W.B.

Saunders Company, 2000 : 520-74.

7. Murer $\mathrm{H}$, Kaissling B, Forster I, Biber J. Cellular mechanisms in proximal tubular handling of phosphate. In: Seldin DW, Giebisch G, eds. The kidney : physiology and pathophysiology, $3^{\mathrm{e}} \mathrm{ed}$. Philadelphie : LippincottWilliams and Wilkins, 2000 : 1869-84.

8. Segawa H, Kaneko I, Takahashi A, et al. Growthrelated renal type $\| \mathrm{Na} / \mathrm{Pi}$ cotransporter. J Biol Chem 2002 (sous presse); publication électronique 5 mars 2002.

\section{Psychologie en néphrologie}

Réalisés par des néphrologues, transplanteurs et une généticienne, des psychiatres, psychologues et une psychanalyste, ces travaux établissent que somaticiens et «psy » peuvent travailler, voire créer ensemble.

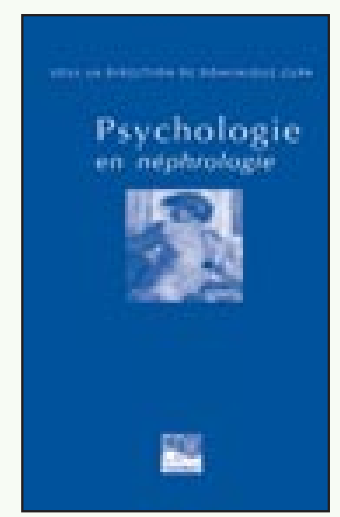

ISBN : 2-84254-043-3 156 pages

\section{Bon de commande}

À retourner à EDK, 10 Villa d'Orléans - 75014 PARIS

Tél. : 0140642749 - Fax : 0140642747 - E-mail : editorial@edk.fr

NOM :

Prénom :

Adresse :

Code postal :

Ville :

Pays :

Fonction :

Je souhaite recevoir l'ouvrage Psychologie en néphrologie : $16 €+3 €$ de port $=\mathbf{1 9} €$ TTC

en exemplaire, soit un total de $€$

$\square$ Par chèque, à l'ordre de $\mathbf{E} \mathbf{D} \mathbf{K}$

Par carte bancaire :

$\square$ Visa

$\square$ Eurocard/Mastercard

American Express

Carte $n^{\circ} \mid \begin{array}{llllllllllllllllll}\mid & \mid & \mid & \mid & \mid & \mid & \mid & \mid & 1 & \mid & \mid & \mid & 1 & \mid & \mid & \mid & \mid\end{array}$ Signature :

Date d'expiration: $\quad \underline{1}|\underline{1}|$ 